\title{
Original
}

\section{PAPEL DE LA RESILIENCIA EN LA EVOLUCIÓN DE LOS PACIENTES CON CÁNCER.}

ROLE OF RESILIENCE IN THE EVOLUTION OF PATIENTS WITH CANCER.

Natividad Calero Mercado ${ }^{1}$, Ana María Manrique Romero², Ana María

Obrero Coronado ${ }^{3}$.

1. Unidad de Medicina Interna, Hospital Valle de los Pedroches, Córdoba, España.

2. Servicio de Urgencias, Hospital Torrecárdenas, Almería, España.

3. Centro de Salud Bujalance, Distrito Sanitario Córdoba Sur, España.

Correspondencia: nati cm 6@hotmail.com

\section{RESUMEN}

Objetivo principal: Analizar los beneficios de la resiliencia en pacientes con cáncer. Metodología: Se realizó una búsqueda bibliográfica en las bases de datos PubMed y Cuiden.

Resultados: Se evidencia que la capacidad de resiliencia es beneficiosa en todo el proceso del cáncer, para ayudar al paciente en todas las dimensiones que verá afectadas (ajuste emocional, emociones positivas -afrontamiento-, evolución del paciente y calidad de vida). Este es un hecho notable, ya que de la forma en que el paciente con cáncer aborde su enfermedad repercutirá en su estado de salud, de forma favorable o desfavorable, dependiendo de la situación o el estado que adopte la persona en esa situación.

Conclusión: Sería conveniente fomentar modelos de resiliencia, ya que de la forma en que el paciente con cáncer aborde su enfermedad repercutirá en su estado de salud.

Palabras clave: "resiliencia", "conductas resilientes", "cáncer" "afrontamiento".

\section{ABSTRACT}

Main objective: To analyze the impact of resilience in patients with cancer. Methodology: A bibliographic search was performed in PubMed and Cuiden databases.

Main results: It is evident that the ability to resilience is beneficial throughout the cancer process, to help the patient in all the dimensions that will be affected (emotional adjustment, positive emotions confrontation-, patient evolution and quality of life). This is a remarkable fact, since the way in which the patient with cancer addresses their illness will affect their health status, in a favorable or unfavorable way, depending on the situation or the state adopted by the person in that situation.

Main conclusion: It would be advisable to promote resilience models, since the way in which the patient with cancer addresses their illness will affect their health status.

Keywords: "resilience", "resilient behaviors", "cancer", "front facing". 


\section{INTRODUCCIÓN}

Tener cáncer es una experiencia vital estresante, traumática y difícil que va a tener importantes repercusiones psicológicas que afectarán a diferentes áreas de la vida del paciente. ${ }^{1}$ Es una de las principales causas de mortalidad y morbilidad en todo el mundo. En 2012 unas 14 millones de personas se vieron afectadas por esta enfermedad y 8,2 millones murieron por dicha causa. Se prevé un aumento del $70 \%$ en los próximos 20 años. $^{2}$

Naturalmente, el diagnóstico de cáncer supone algún tipo de malestar emocional, considerándose normal, en la persona que lo recibe. Éste generalmente padece de un estado de crisis que se manifiesta en un desequilibrio social, físico y psicológico. Viven este suceso traumático con temor, tristeza, aislamiento o preocupación. Lo consideran como sinónimo de dolor y/o muerte próxima. ${ }^{3}$ La comunicación del diagnóstico, las variables de la enfermedad y tratar de asimilar los cambios que ocurren son factores de riesgo que se pueden convertir en un peligro potencial para las personas o en una oportunidad para la propia recuperación y adaptación. ${ }^{4}$

Convertir el diagnóstico del cáncer en un suceso traumático o en una oportunidad para el fortalecimiento depende únicamente del paciente y de la capacidad resiliente que desarrolle en su proceso de curación. ${ }^{5}$ Cuando hablamos de resiliencia nos referimos a la capacidad universal que permite, a las personas, impedir, minimizar - superar los efectos dañinos de la adversidad. La resiliencia está asociada a una serie de factores protectores que la promueven. ${ }^{4}$ Algunas características de la personalidad y del entorno propuestas son: la capacidad de afrontamiento y de resolución de problemas, la seguridad en uno mismo, sentimiento de autosuficiencia, creer que uno puede influir en lo que sucede a su alrededor, etc. amortiguan el impacto de los estresores, alterando o revirtiendo la predicción de resultados negativos. $^{2}$ El concepto de resiliencia incluye dos aspectos relevantes: resistir el suceso y rehacerse del mismo. No basta con una simple recuperación de la situación, ya que ésta implica un retorno gradual hacia la normalidad funcional, sino, además, mantener el equilibrio estable durante todo el proceso. ${ }^{1}$

Entendiendo la resiliencia como, una variable psicológica que potencia un afrontamiento positivo para una adecuada recuperación tanto física como psicológica, nuestro objetivo es analizar cuáles son los beneficios de la resiliencia en pacientes con cáncer.

\section{METODOLOGÍA}

Se realizó una búsqueda en las bases de datos PubMed y Cuiden durante los meses enero y febrero de 2019. Los descriptores utilizados fueron: "resiliencia", "conductas resilientes", "cáncer" y "afrontamiento".

En la tabla 1 se muestran los resultados encontrados en cada base de datos con cada descriptor empleado.

Se establecieron los criterios de inclusión: estudios en inglés y español posteriores al año 2004 que medían la resiliencia y las conductas resilientes de los pacientes para valorar su influencia en las variables psicológicas y físicas, así como la calidad de vida, con acceso libre a texto completo.

\begin{tabular}{|c|c|c|}
\hline & PubMed & Cuiden \\
\hline "resiliencia" & 52 & 94 \\
\hline $\begin{array}{c}\text { "conductas } \\
\text { resilientes" }\end{array}$ & 38 & 13 \\
\hline "cáncer" & 156 & 95 \\
\hline $\begin{array}{c}\text { "afrontamiento" } \\
\text { Total: 479 } \\
\text { artículos }\end{array}$ & 23 & 810 \\
\hline
\end{tabular}

Tabla 1. Descriptores y resultados.

Los criterios de exclusión fueron: aquellos trabajos en los que no se especificaba la 
muestra, los objetivos, el procedimiento ni los resultados, anteriores a 2004, en idioma distinto a inglés o español, y aquellos de los que no se pudo conseguir el texto completo de acceso libre.

Tras aplicar los criterios de inclusión y exclusión, y tras eliminar los duplicados, se obtuvieron 85 artículos, descartándose un total de 309 artículos. En la tabla 2 se clasifican los estudios que cumplían los criterios de inclusión/exclusión para nuestro trabajo en cada base de datos empleada para su búsqueda.

\begin{tabular}{|l|l|}
\hline PubMed & Cuiden \\
\hline 30 & 55 \\
\hline
\end{tabular}

Tabla 2. Estudios con criterios cumplidos.

Posteriormente, se hizo una lectura crítica y se descartaron 70 artículos, quedando para nuestro trabajo 15 trabajos seleccionados. El proceso de revisión bibliográfica y selección de artículos se representa mediante el diagrama de flujo 1.

\section{RESULTADOS}

Podemos enmarcar los beneficios de la resiliencia en pacientes oncológicos en diferentes apartados, que son: ajuste emocional, emociones positivas (afrontamiento), evolución del paciente y calidad de vida, detallados a continuación:

-Ajuste emocional. Protección frente a la depresión: la resiliencia se sitúa en una corriente de psicología positiva y dinámica de fomento de la salud mental y parece una realidad confirmada por el testimonio de muchísimas personas que, aun habiendo vivido un trauma, han conseguido encajarlo y seguir desenvolviendo y viviendo, incluso, en un nivel superior, como si el trauma vivido hubiera desarrollado recursos latentes e insospechados $3,5,6$. Aunque por mucho tiempo las respuestas de resiliencia se han considerado inusuales e incluso patológicas por los expertos, la literatura científica actual demuestra de forma contundente que la resiliencia es una respuesta común y su aparición no indica patología, sino un ajuste saludable a la

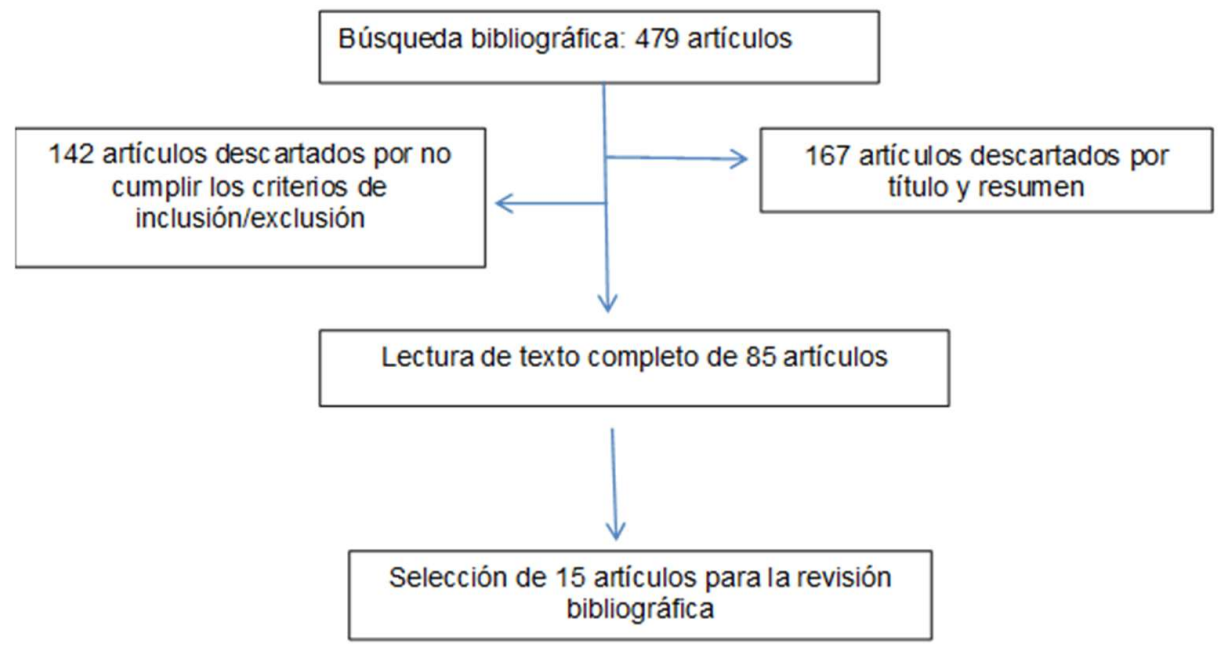

Gráfico 1. Diagrama de flujo de la búsqueda. adversidad $^{1-5}$. Esta parece proteger a las personas frente a la depresión e impulsar su ajuste emocional ${ }^{1,7,8}$. 
-Emociones positivas. Afrontamiento: Las emociones positivas, aunque fenomenológicamente son distintas entre sí, comparten la propiedad de ampliar los repertorios de pensamiento y de acción de las personas y de construir reservas de recursos físicos, intelectuales, psicológicos y sociales disponibles para momentos futuros de crisis. También contribuyen a hacer más resistentes a las personas frente a la adversidad y ayudan a construir resiliencia psicológica ${ }^{3,9,10}$.

Las personas más resilientes suelen experimentar elevados niveles de felicidad y de interés por las cosas en momentos de gran ansiedad provocados experimentalmente. La presencia de emociones positivas durante los momentos de aflicción hace más probable que se desarrollen planes de futuro y estos planes, junto con las emociones positivas, predicen un mejor ajuste psicológico después de haber sufrido un suceso traumático de salud $^{2-4,11}$.

Las emociones positivas parecen ser un ingrediente activo esencial que, además de ayudar a las personas resilientes a no hundirse en la depresión, también contribuye a incrementar sus recursos psicológicos de afrontamiento ${ }^{5,12}$.

-Evolución del paciente: las diferencias encontradas en la evolución de los pacientes con el mismo diagnóstico se deben a varios factores, entre ellos, la forma en que el paciente concibe el diagnóstico, los estilos de afrontamiento que emplea para aceptarlo y la percepción de los recursos sociales, personales y económicos disponibles. ${ }^{1-4}$

La evaluación de estos factores influirá de una forma más positiva o negativa en el paciente, desarrollando así una serie de conductas que, favorecerán o dificultarán, el afrontamiento, adaptación y recuperación de la enfermedad. ${ }^{13}$

Los estudios muestran que las conductas resilientes pueden influir en la recuperación de cualquier enfermedad, especialmente en la oncológica. ${ }^{7,14}$ En los pacientes con cáncer, el optimismo, autocontrol y la habilidad para encontrar significado a las experiencias vividas promueven que el paciente consiga una mejor evolución. ${ }^{8,15}$ Los pacientes que desarrollan estas conductas presentan una personalidad manifestada a través de una alta confianza en sí mismos y una mayor autoestima, están abiertos a nuevas ideas, son más tolerantes y autónomos. ${ }^{6,11}$

- Calidad de vida: El estudio de las emociones positivas y la resiliencia, no solo sirve al ser humano, para la superación de lo que hoy le aqueja, sino más importante aún, le puede ayudar en la prevención de la enfermedad, dándose un cambio radical a lo que generalmente realizamos, como lo es la espera de las manifestaciones del trastorno para empezar a actuar. ${ }^{10-12} \mathrm{De}$ esta manera se obtendrá una mejor calidad de vida, una satisfacción personal que ayude a hacer frente a las adversidades $y$ una mejor aceptación y desarrollo de patologías crónicas o terminales. ${ }^{13-15}$

\section{DISCUSIÓN}

El objetivo de nuestro estudio fue analizar cuáles son los beneficios de la resiliencia en pacientes con cáncer. Los resultados han mostrado que la capacidad de resiliencia es beneficiosa en todo el proceso del cáncer, para ayudar al paciente en todas las dimensiones que verá afectadas (ajuste emocional, emociones positivas afrontamiento-, evolución del paciente y calidad de vida).

De acuerdo a los resultados de nuestro estudio y de otros autores que han investigado previamente sobre nuestra temática, como son Butler, Koopman, Classen y Spiegel, 1999; Devine, Parker, Fouladi y Cohen, 2003; Vinaccia, Quiceno, Fernández y cols., 2005, se constata que la resiliencia es beneficiosa en el paciente con cáncer, incluso algunos de ellos hacen referencia a la atención psicoemocional a los pacientes diagnosticados con cáncer, la 
que puede ser incluida, a criterio del paciente, dentro de los procesos y tratamientos llevados a cabo dentro de la institución especializada donde se atienda, a fin de que se enfoque en la identificación, manejo y expresión emocional, y esté también orientada al trabajo con la familia para mejorar la calidad del soporte social. Algunos autores proponen intervenir en la dinámica familiar para que ésta maximice y conserve una salud integral, de manera que beneficie tanto al paciente como a todos sus miembros y tengan así una mejor preparación al momento de afrontar la enfermedad

Respecto a las limitaciones encontradas a la hora de realizar la presente revisión, hemos encontrado la edad y pronóstico como factores limitantes, ya que podría haber diferencias respecto a la capacidad de resiliencia según la edad, del mismo modo que podría haber diferencias según el pronóstico del paciente. Consideramos una línea de investigación interesante la distinción de estas variables.

\section{CONCLUSIÓN}

Se evidencia que la capacidad de resiliencia es beneficiosa en todo el proceso del cáncer, para ayudar al paciente en todas las dimensiones que verá afectadas (ajuste emocional, emociones positivas afrontamiento-, evolución del paciente y calidad de vida).

Este es un hecho notable, ya que de la forma en que el paciente con cáncer aborde su enfermedad repercutirá en su estado de salud, de forma favorable o desfavorable, dependiendo de la situación o el estado que adopte la persona en esa situación.

Sería conveniente fomentar modelos de resiliencia con la intervención de distintas variables psicológicas y una buena orientación acerca de la enfermedad, proporcionándoles esperanza y calidad de vida.

\section{BIBLIOGRAFÍA}

1. Acinas, M. P. Situaciones traumáticas y resiliencia en personas con cáncer. Psiquiatría Biológica, 2014; 21(2):65-71.

2. Miaja, M.; Moral de la Rubia, J. Significados dados al cáncer y su relación con respuestas psicológicas del duelo en personas tratadas por cáncer. Boletín de Psicología. 2015; 113:7-27.

3. Monteagudo, Y.; Peralta, M. I.; Vicente, A. Estudio de la resiliencia en pacientes oncológicos de la AECC en Castellón. Àgora De Salut III. 2016: 257-265.

4. Álvarez, J.; Cantú, R. Sucesos vitales y factores psicosociales asociados: el caso de pacientes con cáncer. Psicología y Salud. 2011; 21(1):91-102

5. Ascencio, A. Tesis: Resiliencia y estilos de afrontamiento en mujeres con cáncer de mama de un hospital estatal de Chiclayo. Universidad Católica Santo Toribio de Mogrovejo, Chiclayo. 2015.

6. Baider, L. Cáncer y familia: aspectos teóricos y terapéuticos. Revista internacional de psicología Clínica y de la Salud. 2003; 1: 505-520.

7. Fredrickson, B. The role of positive emotions in positive psychology: The broaden-and-built theory of positive emotions, American psychologist. 2001; 56: 218-226.

8. Friedli, L. Mental health, resilience and inequalities. Dinamarca: Organización Mundial de la Salud. 2009.

9. Vázquez C. La ciencia del bienestar psicológico. En Vázquez, A. Hervás, G. Editores. La Ciencia del Bienestar. Madrid, España: Alianza Editorial; 2009; p. 13-43.

10. Ortigosa JM, Méndez FX, Riquelme A. Afrontamiento psicológico de los procedimientos médicos invasivos $y$ dolorosos aplicados para el tratamiento del cáncer infantil y adolescente: La perspectiva cognitivoconductual. Psicooncología. 2009; 6(2-3): 413-28.

11. Keltner B. Walter L. La resiliencia para aquellos que necesitan cuidados de la salud. En: Grotberg E.H. La resiliencia en el mundo de hoy. Barcelona España: Gedisa. 2006; p. 209-34. 
12. Arana, A. Resiliencia: aprendiendo a sobreponerse a la tragedia y a la catástrofe personal. 2010. [acceso 15 marzo 2019]. Disponible en: http://www.psicologiaonline.com/

13. Baider L. Cáncer y familia: aspectos teóricos y terapéuticos. Rev Inter Psicol Clín Salud. 2003; 1(3): 505-20.

14. Siebert, A. La resiliencia. Construir en la adversidad. Barcelona, España: Alienta Editorial; 2007.

15. Garassini ME. Resiliencia y familiares de enfermos oncológicos. Universidad Metropolitana, Venezuela; 2010.

c) (i) $\odot \ominus$ 